\title{
Hypocholesterolemic Effect of Blackcurrant (Ribes nigrum) Extract in Healthy Female Subjects: A Pilot Study
}

\author{
Naoki Nanashima ${ }^{1, *}$,, Kayo Horie ${ }^{1}$, Maiko Kitajima ${ }^{2}$, Shizuka Takamagi ${ }^{2}$, Kasumi Mikami ${ }^{2}$, Naoya In ${ }^{2} \mathbb{C}$ \\ and Toshiko Tomisawa $2, *$ (D) \\ 1 Department of Bioscience and Laboratory Medicine, Hirosaki University Graduate School of Health Sciences, \\ Hirosaki 036-8564, Japan; k-horie@hirosaki-u.ac.jp \\ 2 Department of Nursing Sciences, Hirosaki University Graduate School of Health Sciences, 66-1 Hon-cho, \\ Hirosaki 036-8564, Japan; kitajima@hirosaki-u.ac.jp (M.K.); takamagi@hirosaki-u.ac.jp (S.T.); \\ k-mikami@hirosaki-u.ac.jp (K.M.); in1105@hirosaki-u.ac.jp (N.I.) \\ * Correspondence: nnaoki@hirosaki-u.ac.jp (N.N.); tmtott@hirosaki-u.ac.jp (T.T.)
}

check for updates

Citation: Nanashima, N.; Horie, K.; Kitajima, M.; Takamagi, S.; Mikami,

K.; In, N.; Tomisawa, T.

Hypocholesterolemic Effect of

Blackcurrant (Ribes nigrum) Extract in

Healthy Female Subjects: A Pilot

Study. Molecules 2021, 26, 4085.

https://doi.org/10.3390/

molecules26134085

Academic Editors: Masao Yamasaki and Shaikh Mizanoor Rahman

Received: 15 June 2021

Accepted: 1 July 2021

Published: 4 July 2021

Publisher's Note: MDPI stays neutral with regard to jurisdictional claims in published maps and institutional affiliations.

Copyright: (c) 2021 by the authors. Licensee MDPI, Basel, Switzerland. This article is an open access article distributed under the terms and conditions of the Creative Commons Attribution (CC BY) license (https:/ / creativecommons.org/licenses/by/ $4.0 /)$.

\begin{abstract}
Blackcurrant extract (BCE) ameliorates dyslipidemia in menopausal model animals and in elderly women at a risk of dyslipidemia. However, it is unknown whether the daily intake of BCE can prevent lipid abnormalities in healthy individuals. Lipids are essential for the body, but they also cause arteriosclerosis. In this noncomparative pilot study, we examined the effects of BCE administered for 29 days on serum lipids in young healthy women. Blood samples were collected before and on days 4 and 29 after BCE intake, and 20 lipoprotein fractions in the serum were separated using a gel-permeation high-performance liquid chromatography method to measure the triacylglycerol and cholesterol levels in lipoproteins. There were no effects on lipids on day 4 of BCE intake, but the total cholesterol level decreased on day 29. Furthermore, the levels of total very-low-density lipoprotein (VLDL) cholesterol, small VLDL cholesterol, and large low-density lipoprotein cholesterol were significantly decreased. These results suggest that the daily intake of BCE has a hypocholesterolemic effect in healthy women, and that it is effective in preventing atherosclerosis.
\end{abstract}

Keywords: atherosclerosis; cholesterol; lipoprotein; serum lipid

\section{Introduction}

Blackcurrant (Ribes nigrum L.) is a shrub grown in cooler regions of Europe and New Zealand for its dark-purple berries, which are approximately $1 \mathrm{~cm}$ in diameter. The berries are rich in anthocyanins, a type of polyphenol present in pericarp, and essential fatty acids, such as $\gamma$-linolenic acid, in seeds, which are known to have various healthpromoting functions, including strong antioxidant and phytoestrogenic effects, and blood flow-improving ability [1-4]. Lipids are water-insoluble organic substances found in cells and blood. Several types of lipids are synthesized in the body, but many others are obtained from food. Most of the lipids in human blood bind to proteins, forming water-soluble lipoproteins that flow in the blood. Lipids are metabolized as cell membrane constituents, energy sources, and materials used in the synthesis of hormones and other bioactive compounds. Thus, lipids are essential for the body. However, they have garnered attention as causative agents of arteriosclerosis and cardiovascular diseases [5-8].

The structure of lipoproteins comprises a nucleus made of hydrophobic lipids such as triacylglycerols (TG) and cholesterol esters, which is surrounded by an outer shell of amphipathic lipids such as phospholipids and free cholesterol. Lipoproteins are classified into the following five types according to their specific gravity: chylomicron (CM), very-low-density lipoprotein (VLDL), intermediate-density lipoprotein (IDL), low-density lipoprotein (LDL), and high-density lipoprotein (HDL) $[9,10]$. 
CM is the largest lipoprotein in the blood and carries food-derived TGs. VLDL is a TG-rich lipoprotein that is synthesized in the liver. It becomes an IDL under the action of lipoprotein lipase. The hepatic triglyceride lipase converts most of the IDLs to LDLs, which are taken up by the LDL receptor and catabolized. LDL is the major carrier of cholesterol. In addition, it is the final metabolite of VLDL and is taken up by the LDL receptor present in hepatocytes and peripheral tissues. HDL extracts cholesterol from various tissues and transports it to the liver $[11,12]$.

Polyphenols present in some plants are known to ameliorate dyslipidemia $[13,14]$. It has been reported that the administration of blackcurrant extract (BCE) to animals fed a high-fat diet improved dyslipidemia, and that BCE even lowers serum lipids in humans at risk of dyslipidemia [15-18]. Previously, we showed that BCE is effective in improving menopausal dyslipidemia [19]. This suggests that the daily intake of BCE in healthy subjects may prevent dyslipidemia. However, the effects of BCE on serum lipids in healthy humans have not been investigated. Moreover, a detailed study to examine its effect on 20 lipoprotein fractions separated from serum using a gel-permeation (GP) high-performance liquid chromatography (HPLC) method has not been undertaken. Therefore, we conducted this noncomparative pilot study to investigate the effects of BCE, administered to healthy women without dyslipidemia for approximately 1 month, on serum lipids.

\section{Results}

\subsection{Serum Triglyceride and Total Cholesterol Content}

Blood was collected before and 4 and 29 days after the intake of BCE, to separate serum. The levels of TG and total cholesterol in the serum were measured. Triglyceride levels did not change before and after the intake of BCE (Figure 1A). On the contrary, the total cholesterol level was $172.8 \pm 20.6 \mathrm{mg} / \mathrm{dL}$ before BCE intake. This did not change 4 days after BCE intake, but significantly decreased to $161.9 \pm 25.5 \mathrm{mg} / \mathrm{dL} 29$ days after the intake (Figure 1B).
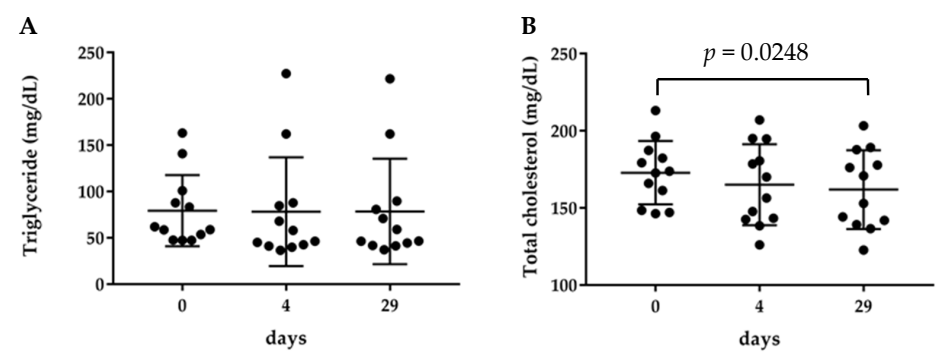

Figure 1. Changes in the levels of serum lipids. Level of (A) triglyceride and (B) total cholesterol. The data are presented as individual data with median and range, $n=12$.

\subsection{Lipoprotein Profiling}

The total cholesterol in the serum decreased 29 days after the intake of BCE; therefore, we investigated the levels of cholesterols in lipoproteins. There were no differences between the levels of CM and HDL cholesterols. The LDL cholesterol level tended to decrease on days 4 and 29 after BCE intake, but no significant difference was observed. However, the VLDL cholesterol level significantly decreased from $24.4 \pm 7.2 \mathrm{mg}$ to $18.6 \pm 4.7 \mathrm{mg}$ after the intake of BCE (Table 1). In contrast, the TG level did not change with any lipoprotein (Supplementary Materials, Table S1). 
Table 1. Cholesterol concentration in the major classes of lipoproteins.

\begin{tabular}{cccc}
\hline Major Class & 0 Day $(\mathbf{m g} / \mathbf{d L})$ & 4 Days $(\mathbf{m g} / \mathbf{d L})$ & 29 Days $(\mathbf{m g} / \mathbf{d L})$ \\
\hline CM & $0.52 \pm 0.3$ & $0.57 \pm 0.8$ & $0.80 \pm 1.0$ \\
VLDL & $24.4 \pm 7.2$ & $21.4 \pm 6.8$ & $18.6 \pm 4.7^{*}$ \\
LDL & $87.9 \pm 12.3$ & $83.5 \pm 21.2$ & $82.8 \pm 20.6$ \\
HDL & $60.1 \pm 9.5$ & $59.5 \pm 11.9$ & $58.7 \pm 11.6$ \\
\hline
\end{tabular}

Data are presented as the mean \pm SD for 12 individuals. ${ }^{*} p<0.05$ vs. 0 day.

\subsection{Lipoprotein Detail Profiling}

Lipoproteins were fractionated; cholesterol in each of the 20 fractions was then quantified. The intake of BCE for 29 days significantly reduced the levels of small VLDL and large LDL cholesterols from $6.80 \pm 2.7 \mathrm{mg}$ to $3.72 \pm 0.7 \mathrm{mg}$ and from $28.1 \pm 8.0 \mathrm{mg}$ to $20.8 \pm 4.5 \mathrm{mg}$, respectively (Table 2). In contrast, the TG level did not change with any lipoprotein (Supplementary Materials, Table S2).

Table 2. Cholesterol concentration in lipoprotein subclasses.

\begin{tabular}{cccc}
\hline Subclass & 0 Day $(\mathbf{m g} / \mathbf{d L})$ & 4 Days $(\mathbf{m g} / \mathbf{d L})$ & 29 Days $(\mathbf{m g} / \mathbf{d L})$ \\
\hline Large VLDL & $12.5 \pm 4.4$ & $11.3 \pm 5.3$ & $11.2 \pm 4.3$ \\
Medium VLDL & $5.04 \pm 1.5$ & $4.16 \pm 1.3$ & $4.66 \pm 1.4$ \\
Small VLDL & $6.80 \pm 2.7$ & $5.92 \pm 1.0$ & $3.72 \pm 0.7^{*}$ \\
Large LDL & $28.1 \pm 8.0$ & $26.8 \pm 6.0$ & $20.8 \pm 4.5^{*}$ \\
Medium LDL & $38.1 \pm 5.3$ & $36.9 \pm 9.6$ & $38.3 \pm 9.6$ \\
Small LDL & $15.0 \pm 4.6$ & $13.7 \pm 3.8$ & $17.1 \pm 4.8$ \\
Very small LDL & $6.79 \pm 2.1$ & $6.15 \pm 1.5$ & $6.61 \pm 1.7$ \\
Very large HDL & $4.86 \pm 2.4$ & $4.86 \pm 2.6$ & $4.07 \pm 2.2$ \\
Large HDL & $18.9 \pm 6.9$ & $18.7 \pm 8.2$ & $18.0 \pm 7.9$ \\
Medium HDL & $20.5 \pm 2.2$ & $20.0 \pm 2.3$ & $20.7 \pm 2.4$ \\
Small HDL & $10.9 \pm 1.5$ & $11.1 \pm 1.5$ & $11.3 \pm 1.7$ \\
Very small HDL & $4.91 \pm 0.7$ & $4.88 \pm 0.7$ & $4.59 \pm 0.6$ \\
\hline
\end{tabular}

Data are presented as the mean \pm SD for 12 individuals. ${ }^{*} p<0.05$ vs. 0 day.

\section{Discussion}

In the present study, we examined the effects of $\mathrm{BCE}$ on serum lipids in young healthy subjects. BCE intake did not change the serum TG level, but significantly decreased the total cholesterol level. Polyphenols such as anthocyanins have been reported to exhibit hypocholesterolemic effects [17,20-23]. Furthermore, Benn et al. reported that BCE powder containing $25 \%$ anthocyanins and $40 \%$ polyphenols reduced the serum levels of total cholesterol, but not of TGs, in mice fed a high-fat diet [15]. Our results are in agreement with those of the previous study. In this study, it is not clear why serum triglyceride levels did not change. Considering that the administration of BCE did not significantly reduce serum triglyceride levels, even in mice fed a high-fat diet, BCE may not be very effective in the absorption of triglycerides [15]. However, because it has been reported that the intake of BCE reduces the amount of triglyceride synthesized in the liver [15,19], it is possible that the synthesis of triglycerides in the liver is suppressed even in healthy subjects.

Regarding the mechanism of BCE-induced hypocholesterolemic effects, Kim et al. reported that anthocyanins in BCE increased the expression of LDL receptors in Caco-2 cells, resulting in a decrease in serum LDL cholesterol levels [24]. In the present study, the intake of BCE possibly increased the uptake of LDL by the LDL receptors; however, because the subjects in this study were young and healthy, the change in LDL cholesterol may have been difficult to detect. However, in the detailed profile of 20 fractions, the levels of small VLDL and large LDL cholesterols were significantly decreased, and it was expected that the serum level of total cholesterol would be lowered due to the enhanced cellular uptake of these lipoproteins. In vitro validation may be necessary in the future.

Polyphenols such as anthocyanins inhibit the absorption of cholesterol from the intestinal tract [25-27]. Furthermore, Nekohashi et al. reported that polyphenols inhibit the 
cholesterol absorption transporter Niemann-Pick C1-Like 1 expressed in human intestinal epithelial cells and prevent the increase in the concentration of food-derived cholesterol in the blood [28]. Although the inhibition of cholesterol absorption was not examined in this study, the inhibitory effect of anthocyanins on cholesterol has been reported [25-27], and it is highly possible that the $\mathrm{BCE}$, being rich in anthocyanins, inhibited the absorption.

More cholesterol is synthesized in the body than is absorbed from food. Hydroxymethylglutaryl-CoA reductase (HMGR) is known to catalyze the rate-limiting step. Several polyphenols are known to inhibit HMG-CoA reductase, and the expression of HMGR is inhibited by BCE and blueberry anthocyanins [24,29]. Based on these reports, it is possible that the expression of HMGR was suppressed by the absorbed anthocyanins in this study.

Among LDL cholesterol, small, dense LDL cholesterol is more likely to be a direct cause of atherosclerosis because it is small enough to penetrate the walls of blood vessels and remains in the blood for a long time to be oxidized [30,31]. In fact, it has been reported that levels of small, dense LDL and very small, dense LDL are increased in patients with coronary artery disease, as determined using the GP-HPLC method in this study [32]. In this study, the VLDL cholesterol level was significantly decreased, and the LDL cholesterol levels tended to decrease. In addition, the levels of small, dense and very small, dense LDL did not decrease, but it was difficult to verify this because the subjects in this study were healthy and the small LDL cholesterol levels were not high at baseline. In this experiment, 12 young healthy subjects were administered BCE for approximately 1 month. In the future, if the study is conducted on a large scale over a longer period, a decrease in small, dense LDL may be observed. Moreover, Tahvonen et al. reported that the administration of blackcurrant seed oil to 15 healthy women for 4 weeks reduced serum LDL cholesterol levels [33]. In this study, the tendency for LDL cholesterol levels to decrease might have been due to the effects of fatty acids present in BCE.

We found that BCE has phytoestrogenic effects. In animal experiments, BCE has been shown to improve lipid abnormalities and vascular endothelial function $[19,34,35]$. In addition, BCE exhibited cosmetic effects in a menopausal rat model [36,37]. In this study, we targeted young, healthy women, but it is predicted that the daily intake of BCE from a young age before menopause will lead to the suppression of arteriosclerosis. These results suggest that the daily intake of BCE may have beneficial effects in women.

\section{Materials and Methods}

\subsection{Materials and Reagents}

The BCE powder, Active Cassis, a dietary supplement, was purchased from Just The Berries Limited, Palmerston North, New Zealand. One hundred grams of the powder consists of $35.0 \mathrm{~g}$ of total anthocyanins, $13.6 \mathrm{mg}$ of carbohydrates, $0.5 \mathrm{~g}$ of fats, and $0.1 \mathrm{~g}$ of proteins, and yields 1650 kilojoules (394 Kcal) of energy [38]. A total of $230 \mathrm{mg} \mathrm{BCE}$ was filled in opaque hard capsules. No adverse events were observed with the use of the BCE powder. The powder is commercially available as a food material. The microbial and heavy metal content of the BCE powder is below the permissible values. Furthermore, the $\mathrm{BCE}$ powder was used within the expiration date, and no safety issues were noted.

\subsection{Subjects}

The subjects in this study were young, healthy females ( $n=12$, mean age $21 \pm 2$ years), students at Hirosaki University. They had typical heights, weights, and body mass index values (Table 3). The exclusion criteria were the presence of any disease, such as hypertension, hyperlipidemia, and diabetes; the use of a weight-reducing dietary regimen; or the consumption of any dietary supplements. All subjects were requested to complete a health and lifestyle questionnaire, and suitable subjects were requested to sign an informed consent form. The study protocol was approved by the Committee of Medical Ethics of Hirosaki University Graduate School of Health Sciences, Hirosaki, Japan (permis- 
sion number: 2016-046 and date of approval: 20 March 2019). All participants provided informed consent.

Table 3. Baseline characteristics of participants $(n=12)$.

\begin{tabular}{cc}
\hline Variable & Value \\
\hline Age, years & $21 \pm 2$ \\
Height, $\mathrm{cm}$ & $158.4 \pm 14.4$ \\
Weight, $\mathrm{kg}$ & $52.9 \pm 17.1$ \\
Body mass index, $\mathrm{kg} / \mathrm{m}^{2}$ & $21.1 \pm 4.1$ \\
\hline
\end{tabular}

Values are expressed as means \pm SD.

\subsection{Study Design}

The subjects were administered two capsules daily for 28 days. Blood was collected one day before BCE intake (day 0) and on days 4 (day 4 ) and 29 (day 29) of BCE intake (Table 4). The blood samples were placed in blood collection tubes and the serum was separated by centrifugation at $1500 \times g$ for $10 \mathrm{~min}$.

Table 4. Test schedule.

\begin{tabular}{ccccccccc}
\hline Days & $\mathbf{0}$ & $\mathbf{1}$ & $\mathbf{2}$ & $\mathbf{3}$ & $\mathbf{4}$ & $\boldsymbol{\cdots}$ & $\mathbf{2 8}$ & $\mathbf{2 9}$ \\
\hline Intake BCE (460 mg/day) & & $\bigcirc$ & $\bigcirc$ & $\bigcirc$ & $\bigcirc$ & $\cdots$ & $\bigcirc$ & \\
\hline Blood sampling & $\bigcirc$ & & & & $\bigcirc$ & $\cdots$ & & $\bigcirc$ \\
\hline
\end{tabular}

\subsection{Biochemical Analysis of Serum Lipids}

The lipoprotein and serum lipid profile data were obtained using GP-HPLC, LipoSEARCH ${ }^{\circledR}$, which is a lipoprotein analysis service; the analysis was performed at Skylight Biotech, Akita, Japan. Briefly, cholesterol and triglycerides contained in the major fractions of lipoproteins (CM, VLDL, LDL, and HDL) and the 20 fractions (subclass defined by particle diameter, Table 5) were determined by GP-HPLC [39]. The major classes of lipoproteins are divided into 20 fractions by diameter. Fractions $1-2$ are CM, fractions 3-7 are VLDL, fractions 8-13 are LDL, and fractions 14-20 are HDL [39]. In addition, each lipoprotein was classified into a subclass, the details of which are shown in Table 5.

\subsection{Statistical Analysis}

Results are expressed as the mean \pm standard deviation. Graphs were generated using GraphPad Prism 7.0 ver. 7.03 (GraphPad Prism, San Diego, CA, USA). Statistically significant differences were determined using a paired one-way analysis of variance (ANOVA) with Bonferroni correction using the bell curve for Excel ver. 3.2 (Social Survey Research Information Co., Ltd., Tokyo, Japan). Results with $p$-values $<0.05$ were considered statistically significant. 
Table 5. Particle diameter in major classes and subclasses of lipoproteins.

\begin{tabular}{|c|c|c|c|}
\hline Fraction No. & Particle Diameter (nm) & Subclass & Major Class \\
\hline 1 & $>90$ & - & \multirow{2}{*}{$\mathrm{CM}(>80 \mathrm{~nm})$} \\
\hline 2 & 75.0 & - & \\
\hline 3 & 64.0 & \multirow{3}{*}{ Large VLDL } & \multirow{5}{*}{ VLDL (30-80 nm) } \\
\hline 4 & 53.6 & & \\
\hline 5 & 44.5 & & \\
\hline 6 & 36.8 & Medium VLDL & \\
\hline 7 & 31.3 & Small VLDL & \\
\hline 8 & 28.6 & Large LDL & \multirow{6}{*}{ LDL (16-30 nm) } \\
\hline 9 & 25.5 & Medium LDL & \\
\hline 10 & 23.0 & \multirow{2}{*}{ Small LDL } & \\
\hline 11 & 20.7 & & \\
\hline 12 & 18.6 & \multirow{2}{*}{ Very small LDL } & \\
\hline 13 & 16.7 & & \\
\hline 14 & 15.0 & \multirow{2}{*}{ Very large HDL } & \multirow{7}{*}{ HDL (8-16 nm) } \\
\hline 15 & 13.5 & & \\
\hline 16 & 12.1 & Large HDL & \\
\hline 17 & 10.9 & Medium HDL & \\
\hline 18 & 9.8 & Small HDL & \\
\hline 19 & 8.8 & \multirow{2}{*}{ Very small HDL } & \\
\hline 20 & 7.6 & & \\
\hline
\end{tabular}

\section{Conclusions}

Serum dyslipidemia is a risk factor for atherosclerosis. In this study, we examined the effects of BCE on serum lipid levels in healthy young women as test subjects. There was no effect after 4 days of BCE intake, but the total serum cholesterol level was lowered after 29 days of intake. In addition, the VLDL cholesterol level was significantly lowered, and LDL cholesterol also showed a decreasing trend. It was difficult to detect the effect of LDL cholesterol in this study because the subjects were healthy. BCE inhibits the absorption of cholesterol, suppresses the expression of HMGR, and promotes the uptake of LDL by the LDL receptor; therefore, it is presumed that serum cholesterol decreased as a result of these synergistic effects. Furthermore, because this was a noncomparative and pilot study, it will be necessary to conduct randomized placebo-controlled studies for a longer period and with a larger number of individuals in the future to confirm the results.

Supplementary Materials: The following are available online, Table S1: Triglyceride concentration in major classes of lipoproteins; Table S2: Triglyceride concentration in lipoprotein subclasses.

Author Contributions: N.N., K.H. and T.T. designed the study; N.N., K.H., M.K., S.T., K.M. and N.I. performed the experiments and analyzed the data; N.N. and K.H. acquired funding; N.N. wrote the manuscript; T.T. edited the manuscript. All authors have read and agreed to the published version of the manuscript.

Funding: This research was partially supported by the Japan Society for the Promotion of Science KAKENHI grant number 20K02402 and the Adaptable and Seamless Technology Transfer Program through Target-driven R\&D (A-STEP) from the Japan Science and Technology Agency (JST) grant number JPMJTM19E5.

Institutional Review Board Statement: The study was conducted according to the guidelines of the Declaration of Helsinki and was approved by the Committee of Medical Ethics of Hirosaki University Graduate School of Health Sciences, Hirosaki, Japan (permission number: 2016-046 and date of approval: 20 March 2019). 
Informed Consent Statement: Informed consent was obtained from all subjects involved in the study.

Data Availability Statement: The data that support the findings of this study are available from the corresponding author, upon reasonable request.

Acknowledgments: The authors would like to thank the volunteers who participated in this study.

Conflicts of Interest: The authors declare no conflict of interest.

Sample Availability: Samples of the BCE powder are available from the authors.

\section{References}

1. Cortez, R.E.; Gonzalez de Mejia, E. Blackcurrants (Ribes nigrum): A review on chemistry, processing, and health benefits. J. Food Sci. 2019, 84, 2387-2401. [CrossRef]

2. Gopalan, A.; Reuben, S.C.; Ahmed, S.; Darvesh, A.S.; Hohmann, J.; Bishayee, A. The health benefits of blackcurrants. Food Funct. 2012, 3, 795-809. [CrossRef]

3. Nanashima, N.; Horie, K.; Maeda, H. Phytoestrogenic activity of blackcurrant anthocyanins is partially mediated through estrogen receptor beta. Molecules 2017, 23, 74. [CrossRef] [PubMed]

4. Nanashima, N.; Horie, K.; Tomisawa, T.; Chiba, M.; Nakano, M.; Fujita, T.; Maeda, H.; Kitajima, M.; Takamagi, S.; Uchiyama, D.; et al. Phytoestrogenic activity of blackcurrant (Ribes nigrum) anthocyanins is mediated through estrogen receptor alpha. Mol. Nutr. Food Res. 2015, 59, 2419-2431. [CrossRef] [PubMed]

5. Choy, P.C.; Siow, Y.L.; Mymin, D.; Karim, O. Lipids and atherosclerosis. Biochem. Cell Biol. 2004, 82, 212-224. [CrossRef] [PubMed]

6. Farnier, M.; Zeller, M.; Masson, D.; Cottin, Y. Triglycerides and risk of atherosclerotic cardiovascular disease: An update. Arch. Cardiovasc. Dis. 2021, 114, 132-139. [CrossRef]

7. Kohsaka, S.; Jin, Z.; Rundek, T.; Homma, S.; Sacco, R.L.; Di Tullio, M.R. Relationship between serum lipid values and atherosclerotic burden in the proximal thoracic aorta. Int. J. Stroke 2010, 5, 257-263. [CrossRef]

8. Mortensen, M.B.; Nordestgaard, B.G. Elevated LDL cholesterol and increased risk of myocardial infarction and atherosclerotic cardiovascular disease in individuals aged 70-100 years: A contemporary primary prevention cohort. Lancet 2020, 396, 1644-1652. [CrossRef]

9. Corvilain, B. Lipoprotein metabolism. Rev. Med. Brux. 1997, 18, 3-9. [PubMed]

10. Fielding, C.J. Lipoprotein receptors, plasma cholesterol metabolism, and the regulation of cellular free cholesterol concentration. FASEB J. 1992, 6, 3162-3168. [CrossRef]

11. Kovanen, P.T. Regulation of plasma cholesterol by hepatic low-density lipoprotein receptors. Am. Heart J. 1987, 113, 464-469. [CrossRef]

12. Small, D.M. Mechanisms of reversed cholesterol transport. Agents Actions Suppl. 1988, 26, 135-146.

13. Liu, C.; Sun, J.; Lu, Y.; Bo, Y. Effects of anthocyanin on serum lipids in dyslipidemia patients: A systematic review and meta-analysis. PLoS ONE 2016, 11, e0162089. [CrossRef]

14. Pan, H.; Gao, Y.; Tu, Y. Mechanisms of body weight reduction by black tea polyphenols. Molecules 2016, 21, 1659. [CrossRef] [PubMed]

15. Benn, T.; Kim, B.; Park, Y.K.; Yang, Y.; Pham, T.X.; Ku, C.S.; Farruggia, C.; Harness, E.; Smyth, J.A.; Lee, J.Y. Polyphenol-rich blackcurrant extract exerts hypocholesterolaemic and hypoglycaemic effects in mice fed a diet containing high fat and cholesterol. Br. J. Nutr. 2015, 113, 1697-1703. [CrossRef]

16. Fa-lin, Z.; Zhen-yu, W.; Yan, H.; Tao, Z.; Kang, L. Efficacy of blackcurrant oil soft capsule, a Chinese herbal drug, in hyperlipidemia treatment. Phytother. Res. 2010, 24, S209-S213. [CrossRef]

17. Jurgonski, A.; Juskiewicz, J.; Zdunczyk, Z.; Matusevicius, P.; Kolodziejczyk, K. Polyphenol-rich extract from blackcurrant pomace attenuates the intestinal tract and serum lipid changes induced by a high-fat diet in rabbits. Eur. J. Nutr. 2014, 53, 1603-1613. [CrossRef] [PubMed]

18. Lee, Y.; Pham, T.X.; Bae, M.; Hu, S.; O’Neill, E.; Chun, O.K.; Han, M.J.; Koo, S.I.; Park, Y.K.; Lee, J.Y. Blackcurrant (Ribes nigrum) prevents obesity-induced nonalcoholic steatohepatitis in mice. Obesity 2019, 27, 112-120. [CrossRef]

19. Nanashima, N.; Horie, K.; Yamanouchi, K.; Tomisawa, T.; Kitajima, M.; Oey, I.; Maeda, H. Blackcurrant (Ribes nigrum) extract prevents dyslipidemia and hepatic steatosis in ovariectomized rats. Nutrients 2020, 12, 1541. [CrossRef]

20. Castaldo, L.; Narváez, A.; Izzo, L.; Graziani, G.; Gaspari, A.; Minno, G.D.; Ritieni, A. Red wine consumption and cardiovascular health. Molecules 2019, 24, 3626. [CrossRef]

21. Nagasako-Akazome, Y.; Kanda, T.; Ikeda, M.; Shimasaki, H. Serum cholesterol-lowering effect of apple polyphenols in healthy subjects. J. Oleo. Sci. 2005, 54, 143-151. [CrossRef]

22. Nagasako-Akazome, Y.; Kanda, T.; Ohtake, Y.; Shimasaki, H.; Kobayashi, T. Apple polyphenols influence cholesterol metabolism in healthy subjects with relatively high body mass index. J. Oleo. Sci. 2007, 56, 417-428. [CrossRef] [PubMed]

23. Wang, H.; Liu, D.; Ji, Y.; Liu, Y.; Xu, L.; Guo, Y. Dietary supplementation of black rice anthocyanin extract regulates cholesterol metabolism and improves gut microbiota dysbiosis in C57BL/6J mice fed a high-fat and cholesterol diet. Mol. Nutr. Food Res. 2020, 64, e1900876. [CrossRef] [PubMed] 
24. Kim, B.; Bae, M.; Park, Y.K.; Ma, H.; Yuan, T.; Seeram, N.P.; Lee, J.Y. Blackcurrant anthocyanins stimulated cholesterol transport via post-transcriptional induction of LDL receptor in Caco-2 cells. Eur. J. Nutr. 2018, 57, 405-415. [CrossRef] [PubMed]

25. Chamnansilpa, N.; Aksornchu, P.; Adisakwattana, S.; Thilavech, T.; Makynen, K.; Dahlan, W.; Ngamukote, S. Anthocyanin-rich fraction from Thai berries interferes with the key steps of lipid digestion and cholesterol absorption. Heliyon 2020, 6, e05408. [CrossRef] [PubMed]

26. Thilavech, T.; Adisakwattana, S. Cyanidin-3-rutinoside acts as a natural inhibitor of intestinal lipid digestion and absorption. BMC Complement. Altern. Med. 2019, 19, 242. [CrossRef] [PubMed]

27. Yao, S.L.; Xu, Y.; Zhang, Y.Y.; Lu, Y.H. Black rice and anthocyanins induce inhibition of cholesterol absorption in vitro. Food Funct. 2013, 4, 1602-1608. [CrossRef]

28. Nekohashi, M.; Ogawa, M.; Ogihara, T.; Nakazawa, K.; Kato, H.; Misaka, T.; Abe, K.; Kobayashi, S. Luteolin and quercetin affect the cholesterol absorption mediated by epithelial cholesterol transporter niemann-pick c1-like 1 in caco-2 cells and rats. PLoS ONE 2014, 9, e97901. [CrossRef]

29. Liang, Y.; Chen, J.; Zuo, Y.; Ma, K.Y.; Jiang, Y.; Huang, Y.; Chen, Z.Y. Blueberry anthocyanins at doses of 0.5 and $1 \%$ lowered plasma cholesterol by increasing fecal excretion of acidic and neutral sterols in hamsters fed a cholesterol-enriched diet. Eur. J. Nutr. 2013, 52, 869-875. [CrossRef]

30. Hirayama, S.; Miida, T. Small dense LDL: An emerging risk factor for cardiovascular disease. Clin. Chim. Acta 2012, 414, 215-224. [CrossRef]

31. Ivanova, E.A.; Myasoedova, V.A.; Melnichenko, A.A.; Grechko, A.V.; Orekhov, A.N. Small dense low-density lipoprotein as biomarker for atherosclerotic diseases. Oxid. Med. Cell. Longev. 2017, 2017, 1273042. [CrossRef] [PubMed]

32. Okazaki, M.; Usui, S.; Fukui, A.; Kubota, I.; Tomoike, H. Component analysis of HPLC profiles of unique lipoprotein subclass cholesterols for detection of coronary artery disease. Clin. Chem. 2006, 52, 2049-2053. [CrossRef]

33. Tahvonen, R.L.; Schwab, U.S.; Linderborg, K.M.; Mykkanen, H.M.; Kallio, H.P. Black currant seed oil and fish oil supplements differ in their effects on fatty acid profiles of plasma lipids, and concentrations of serum total and lipoprotein lipids, plasma glucose and insulin. J. Nutr. Biochem. 2005, 16, 353-359. [CrossRef]

34. Horie, K.; Nanashima, N.; Maeda, H. Phytoestrogenic effects of blackcurrant anthocyanins increased endothelial nitric oxide synthase (eNOS) expression in human endothelial cells and ovariectomized rats. Molecules 2019, 24, 1259. [CrossRef] [PubMed]

35. Horie, K.; Nanashima, N.; Maeda, H.; Tomisawa, T.; Oey, I. Blackcurrant (Ribes nigrum L.) extract exerts potential vasculoprotective effects in ovariectomized rats, including prevention of elastin degradation and pathological vascular remodeling. Nutrients 2021, 13, 560. [CrossRef]

36. Nanashima, N.; Horie, K. Blackcurrant extract with phytoestrogen activity alleviates hair loss in ovariectomized rats. Molecules 2019, 24, 1272. [CrossRef]

37. Nanashima, N.; Horie, K.; Maeda, H.; Tomisawa, T.; Kitajima, M.; Nakamura, T. Blackcurrant anthocyanins increase the levels of collagen, elastin, and hyaluronic acid in human skin fibroblasts and ovariectomized rats. Nutrients 2018, 10, 495. [CrossRef] [PubMed]

38. Yoshizaki, A.; Tomisawa, T.; Osanai, T.; Nanashima, N.; Kitajima, M.; Mikami, K.; Fujita, T.; Maeda, H.; Kato, Y. Single oral administration of anthocyanin rescues smoking-induced dysfunction in young smokers but facilitates oxidative stress in non-smokers. Food Nutr. Sci. 2018, 9, 179-190. [CrossRef]

39. Toshima, G.; Iwama, Y.; Kimura, F.; Matsumoto, Y.; Miura, M.; Takahashi, J.; Yasuda, H.; Arai, N.; Mizutani, H.; Hata, K.; et al. LipoSEARCH ${ }^{\circledR}$; Analytical GP-HPLC method for lipoprotein profiling and its applications. J. Biol. Macromol. 2013, 13, 21-32. 